\title{
Promoção das Habilidades Sociais de Futuros Professores com foco na
}

\section{Educação Inclusiva}

\author{
Patricia Lorena Quiterio* \\ Universidade do Estado do Rio de Janeiro - UERJ, Rio de Janeiro, RJ, Brasil \\ ORCID: http://orcid.org/0000-0002-4553-6429 \\ Leila Regina d'Oliveira de Paula Nunes** \\ Universidade do Estado do Rio de Janeiro - UERJ, Rio de Janeiro, RJ, Brasil \\ ORCID: http://orcid.org/0000-0003-2012-6973 \\ Bruna de Lima Camelo*** \\ Universidade do Estado do Rio de Janeiro - UERJ, Rio de Janeiro, RJ, Brasil \\ ORCID: http://orcid.org/0000-0002-6358-9102 \\ Jenniffer Pires da Silva**** \\ Universidade do Estado do Rio de Janeiro - UERJ, Rio de Janeiro, RJ, Brasil \\ ORCID: http://orcid.org/0000-0002-9172-7744 \\ Marwin Machay Indio do Brasil do Carmo***** \\ Universidade do Estado do Rio de Janeiro - UERJ, Rio de Janeiro, RJ, Brasil \\ ORCID: http://orcid.org/0000-0002-1052-6300
}

\begin{abstract}
RESUMO
O desenvolvimento das habilidades sociais de futuros professores é fator essencial para a inclusão de alunos com deficiência. O objetivo deste estudo foi avaliar os efeitos de um Programa de Promoção de Habilidades Sociais com foco na inclusão, voltado para estudantes universitários do curso de Pedagogia. Participaram 10 estudantes universitárias (idade entre 18 e 56 anos). Os instrumentos de avaliação foram: (a) Inventário de Habilidades Sociais (pré, Pós-intervenção e seguimento), (b) Questionário Diagnóstico (pré e Pós-intervenção) e (c) Diário de Campo (processual). Os escores de Pós-intervenção do Inventário de Habilidades Sociais foram significativamente maiores do que os escores pré-intervenção e comparações post hoc indicaram médias maiores, estatisticamente significantes, para os escores no seguimento, em relação à pré-intervenção, no Escore Total. A análise do questionário diagnóstico constatou maior compreensão do papel do interlocutor e ampliação dos conceitos teóricos e sua aplicabilidade. As vivências e dinâmicas evidenciaram o desenvolvimento das subclasses de habilidades sociais das graduandas, especialmente da assertividade e da empatia. Os escores obtidos no seguimento revelaram que a melhora nos fatores de habilidades sociais se manteve no intervalo de dois anos, indicando que as futuras professoras desenvolveram de modo adequado as habilidades sociais, contribuindo para sua atuação no contexto educativo.
\end{abstract}

Palavras-chave: habilidades sociais, intervenção, estudantes universitários, inclusão, avaliação de programa. 


\title{
Promoting the Social Skills of Future Teachers focusing on Inclusive
}

\section{Education}

\begin{abstract}
The development of future teachers' social skills is an essential factor for the inclusion of students with disabilities. The aim of this study was to evaluate the effects of a Social Skills Promotion Program with focus on inclusion, for university students in the Pedagogy course. Ten university students (aged between 18 and 56 years) participated. The assessment instruments were: (a) Social Skills Inventory (pre, post-intervention and follow-up), (b) Diagnostic Questionnaire (pre and post-intervention) and, (c) Field Diary (procedural). The post-intervention scores for the Social Skills Inventory were significantly higher than the preintervention scores and post hoc comparisons indicated statistically significant higher means for the scores in the follow-up, compared to the pre-intervention, in the Total Score. The analysis of the diagnostic questionnaire showed a higher comprehension about the role of the interlocutor and an expansion of theoretical concepts and its applicability. The experiences and dynamics evidenced a development of subclasses of social skills, especially assertiveness and empathy. The scores obtained in the follow-up revealed that the improvement in social skills factors was maintained over a two-year interval, indicating that future teachers developed social skills in an appropriate manner, contributing to their performance in the educational context.
\end{abstract}

Keywords: social skills, intervention, university students, inclusion, program evaluation.

\section{Promoviendo de las Habilidades Sociales de los Futuros Maestros con}

\section{enfoque en la Educación Inclusiva}

\section{RESUMEN}

El desarrollo de las habilidades sociales de los futuros docentes es un factor fundamental para la inclusión de estudiantes con discapacidad. El objetivo de este estudio fue evaluar los efectos de un Programa de Promoción de Habilidades Sociales con enfoque de inclusión, dirigido a estudiantes universitarios de la carrera de Pedagogía. Participaron diez estudiantes universitarias (edad entre 18 y 56 años). Los instrumentos de evaluación fueron: (a) Inventario de Habilidades Sociales (pre, posintervención y seguimiento), (b) Cuestionario de Diagnóstico (pre y posintervención) y (c) Diario de Campo (procedimental). Los puntajes posteriores a la intervención del Inventario de Habilidades Sociales fueron significativamente más altos que los puntajes previos a la intervención y las comparaciones post hoc indicaron promedios más altos, estadísticamente significativos, para los puntajes de seguimiento, en comparación con la preintervención, en el Puntaje Total. El análisis del cuestionario de diagnóstico encontró una mayor comprensión del rol del interlocutor y una ampliación de los conceptos teóricos y su aplicabilidad. Las experiencias y dinámicas mostraron el desarrollo de las subclases de habilidades sociales de las estudiantes de pregrado, especialmente la asertividad y la empatía. Las puntuaciones obtenidas en el seguimiento revelaron que la mejora en los factores de habilidades sociales se mantuvo en el intervalo de dos años, lo que indica que las futuras docentes desarrollaron las habilidades sociales de manera adecuada, contribuyendo a su desempeño en el contexto educativo. 
Patricia Lorena Quiterio, Leila Regina d'Oliveira de Paula Nunes, Bruna de Lima Camelo, Jenniffer Pires da Silva, Marwin Machay Indio do Brasil do Carmo

Palabras clave: habilidades sociales, intervención, estudiantes universitarios, inclusión, evaluación del programa.

A qualidade das relações interpessoais de um indivíduo impacta diretamente seu desenvolvimento. As Habilidades Sociais (HS) assumem papel de destaque nessas relações, sendo definidas como um conjunto de comportamentos sociais desejáveis, voltados para o enfrentamento de demandas sociais. São atitudes valorizadas pela cultura e apresentam benefícios para o indivíduo, seu grupo e sociedade. São adquiridas e moduladas a partir da convivência e relacionamento com o outro (A. Del Prette \& Z. A. P. Del Prette, 2017).

Eventualmente, o indivíduo dispõe de um repertório de HS, mas não apresenta desempenho considerado socialmente competente. Essa dimensão avaliativa designa o conceito de Competência Social (CS), que se expressa no desempenho, enquanto comportamento observável (A. Del Prette \& Z. A. P. Del Prette, 2017). Tal avaliação do desempenho social apresenta uma funcionalidade, segundo a qual se observa a repercussão deste comportamento no ambiente, analisando o alcance dos objetivos, a ampliação das relações interpessoais e a melhoria da autoestima (Z. A. P. Del Prette \& A. Del Prette, 2012).

A intervenção em HS ocorre por meio de Programas de Promoção das Habilidades Sociais (PPHS), eficazes para promover comportamentos pró-sociais (Wolstencroft, Robinson, Srinivasan, Kerry, Mandy, \& Skuse, 2018). Os PPHS podem ser definidos como o conjunto de técnicas e atividades que visam a estruturação de processos de aprendizagem para aquisição e aperfeiçoamento das HS, bem como dos requisitos da CS (A. Del Prette \& Z. A. P. Del Prette, 2017). Programas de intervenção com foco na prevenção e na saúde mental têm sido desenvolvidos com alunos de educação infantil, familiares, professores da rede pública de ensino, adolescentes, professores e estudantes universitários (Bolsoni-Silva, Leme, Lima, Junior, \& Correia, 2009; Cintra, 2018; DiPerna, Lei, Cheng, Hart, \& Bellinger, 2017; Ferreira, Oliveira, \& Vandenberghe, 2014; Lopes, Dascanio, Ferreira, Z. A. P. Del Prette, \& A. Del Prette, 2017; Vieira-Santos, Z. A. P. Del Prette, \& A. Del Prette, 2018).

Avaliações de programas podem ser realizadas em quatro fases: (a) pré intervenção, (b) avaliação de processo, (c) pós intervenção e, (d) seguimento (A. Del Prette \& Z. A. P. Del Prette, 2017). Segundo Linder (2008), uma crítica comum ao treinamento de HS é a falta de estudos de acompanhamento para investigar se os efeitos de tal formação são generalizados para interações com outros parceiros e contextos, embora novas estratégias estejam sendo 
mais amplamente implementadas para esse objetivo (Vila, 2019). Neste contexto, o papel do facilitador é fundamental enquanto posição técnica, relacionada ao conhecimento das HS e, no caso de pessoas com deficiência, da Comunicação Alternativa (CA) - área multidisciplinar que oferece recursos e estratégias de comunicação, bem como destaca o papel do interlocutor na interação com pessoas com deficiência sem fala articulada (Quiterio \& Nunes, 2013), - e da posição ética, enquanto respeito às diferenças favorecendo os princípios de solidariedade, tolerância e respeito mútuo.

Por mais que no âmbito escolar o professor possua papel fundamental como mediador no desenvolvimento das HS (Bolsoni-Silva, Mariano, Loureiro, \& Bonaccorsi, 2013; Esteves, 2018; Reis, Prata, \& Soares, 2012; Rosin-Pinola, Marturano, Elias, \& Del Prette, 2017) junto à população com ou sem deficiência, a literatura revela que os educadores não aproveitam as situações naturais para promover as interações sociais, menos ainda, elaboram vivências semiestruturadas que envolvam a participação de todos os alunos. Por fim, constata-se que a criança sem deficiência não apresenta iniciativa de contato com a criança com deficiência, que por sua vez não demonstra iniciativa nas relações interpessoais (Manolio, 2009; Rosa \& Menezes, 2019; Rosin-Pinola \& Del Prette, 2014).

Para lidar com essas e outras demandas do contexto educacional, o educador precisa desenvolver as Habilidades Sociais Educativas (HSE). Essas habilidades "são aquelas intencionalmente voltadas para a promoção do desenvolvimento e da aprendizagem do outro, em situação formal ou informal” (Z. A. P. Del Prette \& A. Del Prette, 2007, p. 95). Este papel envolve um modelo de mediação e interação competente, tanto em relação ao objeto de conhecimento como nas práticas interpessoais. Três habilidades referentes às HSE de professores se destacam: (a) planejar, estruturar, apresentar e conduzir atividade interativa; (b) avaliar atividade e desempenhos específicos e; (c) cultivar afetividade e participação dos alunos (A. Del Prette \& Z. A. P. Del Prette, 2017).

Diante desse cenário, estudos têm surgido em âmbito nacional, com a proposta de desenvolver programas de intervenção voltados para professores (Vieira-Santos et al., 2018). Manolio (2009) e Rosa e Menezes (2019) revelaram dificuldade desse público em alterar atitudes em sala de aula. Deste modo, evidencia-se a relevância da intervenção na formação inicial como um diferencial no desenvolvimento das HSE do professor (Deimling, 2013; Quiterio \& Nunes, 2017). Estudos atuais indicam direções para a mediação competente do agente educativo, revelando a necessidade de criatividade para conceber/organizar interações entre os alunos; flexibilidade para mudar o curso do planejamento de ensino; observação e análise dos dados; estratégias de solução de problemas, novas tecnologias, novos desafios e 
Patricia Lorena Quiterio, Leila Regina d'Oliveira de Paula Nunes, Bruna de Lima Camelo, Jenniffer Pires da Silva, Marwin Machay Indio do Brasil do Carmo

aplicação prática dos conhecimentos teóricos (Chaves, Galinha, \& Gontijo, 2017; Cintra, 2018; Rosin-Pinola \& Del Prette, 2014).

Tendo em vista os aspectos anteriormente considerados, promover HS e HSE de estudantes universitários da área da Pedagogia torna-se um importante foco de interesse, pois pode facilitar as relações interpessoais no ambiente escolar, além de fornecer subsídios e estratégias para lidar com diferentes aspectos, englobando a inclusão escolar. No contexto universitário, Leme, Z. A. P. Del Prette e A. Del Prette (2016) ao realizarem uma pesquisa de estado da arte, analisaram 13 artigos de intervenções junto a futuros psicólogos e concluíram que a maior parte ressaltou a caracterização/avaliação de HS. Os autores sinalizam uma lacuna em relação a estudos de promoção de HS do estudante de Psicologia. Ainda no contexto universitário, A. Del Prette e Z. A. P. Del Prette (2003) desenvolveram um PPHS com dez estudantes do último período da graduação em Ciências Exatas. A maioria dos participantes obteve avanço significativo entre os escores pré e Pós-intervenção. Estudo realizado por Chaves et al. (2017) investigou a formação docente do curso de licenciatura em Letras/Espanhol em relação às HS fundamentais à atuação do professor. Os resultados indicaram a necessidade de desenvolvimento dessas habilidades. Os autores propõem o desenvolvimento de uma intervenção considerando a formação integral (aluno e professor) e o papel social do educador.

Observa-se a escassez de intervenções voltadas para a formação inicial de futuros professores tanto em relação às HS quanto às HSE. Apesar da importância deste profissional como mediador nas relações interpessoais construídas em sala de aula (Manolio, 2009; Merritt, Wanless, Rimm-Kauffman, \& Cameron, 2012; Reis et al., 2012; Rosin-Pinola \& Del Prette, 2014) parece que nenhum programa de PPHS com universitários voltou-se para a área de Pedagogia, na qual os universitários, em breve, estarão atuando em salas de aula inclusivas. Considerando isto, desenvolver PPHS com universitários da área de Educação torna-se relevante, pois pode evitar futuras dificuldades interpessoais, bem como favorecer o ingresso no mercado de trabalho.

Considerando os achados da literatura, os estudos citados salientam a importância da contribuição do construto teórico-prático das habilidades sociais à Educação, especialmente ao considerar a formação inicial e continuada do professor, o uso de recursos e estratégias diferenciadas e as relações interpessoais no contexto educativo. No entanto, visualizando o contexto da educação inclusiva, a presente pesquisa buscou minimizar lacunas nos estudos brasileiros, como a avaliação do repertório de HS de futuros professores e o desenvolvimento de um programa de promoção das HS que, além de promover essas habilidades, também 
influenciasse diretamente à ampliação das HSE no contexto inclusivo, inclusive, discutindo sobre a necessidade de o professor adicionar intencionalmente, em seu plano de aula, atividades de promoção das habilidades sociais. O PPHS com estudantes universitários de Pedagogia objetiva desenvolver as HS de futuros professores por meio da automonitoria, do autoconhecimento e dos valores de convivência que, consequentemente, podem propiciar o desenvolvimento dessas habilidades em seus alunos, além de desenvolver suas HSE no manejo adequado da sala de aula, por meio do planejamento e condução de atividade interativa, do estímulo à participação dos alunos, de estratégias de solução de problemas, da experimentação de trabalho em grupo e do fortalecimento de um ambiente saudável, com o intuito de favorecer a saúde mental e o bem-estar. Assim, este artigo descreve os efeitos de uma intervenção exploratória em um grupo de estudantes universitárias de Pedagogia.

\section{Método}

\section{Participantes}

O estudo com caráter exploratório, metodologia de análise quantitativa e qualitativa, seguiu um delineamento quase experimental. A intervenção foi realizada com aplicação de medidas de avaliação pré-intervenção, processual, Pós-intervenção e seguimento. Participaram 10 estudantes universitários do sexo feminino, com idade entre 18 e 56 anos ( $M$ $=24,7 ; d p=10,6)$, matriculadas no terceiro $(30 \%)$ e quarto $(70 \%)$ períodos do curso de Pedagogia em uma universidade pública do Rio de Janeiro. Uma aluna era casada com filhos e as demais solteiras. A classificação socioeconômica das participantes era com prevalência na faixa B2 $(40 \%)$.

\section{Instrumentos}

1. Questionário Diagnóstico (Pré- e Pós-intervenção). Instrumento desenvolvido para esse estudo pela primeira autora. Tem como objetivo verificar o conhecimento sobre CA, HS e HSE, composto pelas seguintes questões: (i) Escreva o que você sabe sobre Comunicação Alternativa (CA), (ii) O que você entende ou já ouviu falar sobre Habilidades Sociais (HS)? Escreva aqui, (iii) Você já ouviu as expressões “desempenho social” e "competência social”? O que você acha que elas significam?, (iv) Você considera importante entender e desenvolver as habilidades sociais educativas para relacionar-se com pessoas com deficiência? Por quê? e, 
(v) Leia esta frase: "Na ausência do outro, o homem não se constrói homem" (Vygotsky). Escreva o que ela significa para você.

\section{Inventário de Habilidades Sociais (IHS-Del Prette, 2011) (Pré, Pós-intervenção e}

Seguimento). A intervenção ocorreu previamente à publicação do instrumento IHS2-Del Prette, por isso foi utilizada a versão anterior. Instrumento de autorrelato para avaliação de HS, composto de 38 itens, cada um descrevendo uma situação de relação interpessoal e uma demanda de habilidade para reagir àquela situação. $\mathrm{O}$ respondente deve estimar a frequência com que reage da forma sugerida em cada item, considerando o total de vezes que se encontrou na situação descrita, e estimar a frequência de sua resposta em escala Likert, com cinco pontos, variando de 0 (nunca ou raramente) a 4 (sempre ou quase sempre). O IHS-DelPrette produz um escore geral, referenciado à norma em termos de percentis, e escores em cinco subescalas de HS: F1 - Enfrentamento e Autoafirmação com Risco; F2 - Autoafirmação na Expressão de Sentimento Positivo; F3 - Conversação e Desenvoltura Social, F4 Autoexposição a Desconhecidos e Situações Novas, e F5 - Autocontrole da Agressividade.

3. Diário de Campo (Avaliação Processual). Instrumento preenchido por uma bolsista de pesquisa para registrar as etapas da intervenção. Tem o objetivo de auxiliar na avaliação das principais mudanças ocorridas durante o programa e, portanto, do impacto da intervenção.

\section{Procedimentos}

O estudo foi aprovado pela Comissão de Ética em Pesquisa de uma Instituição de Ensino Superior (Parecer COEP 004 / 047.3.2011). Todos os participantes assinaram o Termo de Consentimento Livre e Esclarecido (TCLE). Todas as vivências e dinâmicas realizadas durante o PPHS foram videografadas e transcritas por duas alunas do curso de Pedagogia e assistentes de pesquisa.

Coleta de Dados. Em cada encontro havia uma bolsista de pesquisa que redigia o diário de campo e fazia filmagens e fotografias do grupo, especialmente das dinâmicas e vivências, com a função de avaliar o processo de intervenção. Na fase pré-intervenção, informou-se o objetivo do PPHS aos participantes e solicitou-se que respondessem aos dois instrumentos: questionário diagnóstico e IHS-Del Prette. No último encontro, imediatamente após o encerramento do PPHS, as participantes responderam aos mesmos instrumentos e, por fim, dois anos após o encerramento do programa responderam ao IHS-Del Prette, a fim de avaliar a continuidade dos efeitos da intervenção realizada. 
Programa de Promoção das Habilidades Sociais (PPHS). O curso teve a duração de 44h, sendo distribuídas em 22 encontros de duas horas. O formato teórico do curso envolveu discussão de artigos e capítulos de livros sobre HS e, mais especificamente de trabalhos relacionados a pessoas com deficiência. No formato prático, as alunas participaram de vivências, dinâmicas e elaboração de atividades com recursos da CA para posterior intervenção junto a alunos sem fala articulada. Dentre as vivências e dinâmicas realizadas exemplificam-se: (a) primeiro encontro - vivência com foco em elementos da ansiedade, (b) terceiro encontro - vivência "Uma viagem à lua" (Weber, 2011) que visa trabalhar a integração, habilidade para ouvir, clareza na comunicação e bom humor, (c) quarto encontro vivência: "Corredor brasileiro" (Z. A. P. Del Prette \& A. Del Prette, 2007), adaptada para este PPHS, na qual cada graduanda precisava avaliar qual a subclasse de habilidade social que mais precisa desenvolver, (d) sétimo encontro - atividade com foco nas HSE. Cada graduanda recebeu uma frase que trazia comentários negativos sobre seu desempenho e tinham que marcar em uma folha a emoção que a frase lhe despertou. Na segunda etapa da atividade, as alunas receberam o par da sua frase, sendo agora uma frase socialmente habilidosa, (e) nono encontro - atividade "Olhos Vendados" - as graduandas receberam um número, a partir do qual deveriam assumir lugar numa fila em ordem crescente, mas com olhos vendados e sem utilizar a linguagem verbal e, (f) décimo encontro - vivência "Reconhecendo e comunicando emoções” (Z. A. P. Del Prette \& A. Del Prette, 2007), adaptada para este PPHS, na qual uma participante fazia uma expressão facial e corporal com os olhos vendados e o grupo tinha que descobrir a emoção representada.

O programa foi desenvolvido com a seguinte estrutura: (1) revisão da tarefa de casa; (2) dinâmica ou vivência; (3) exposição didática dos conceitos teóricos; (4) treino de habilidades (atividade, dinâmica ou vivência); (5) tarefa de casa (Quiterio \& Nunes, 2017). Uma breve descrição do programa por encontros: (1) apresentação da proposta do curso, com cronograma e lista de tarefas de casa, assinatura do Termo de Consentimento Livre e Esclarecido, aplicação do IHS-Del-Prette e do questionário diagnóstico sobre HS; (2) Conceitos básicos na área de HS, (3 e 4) Subclasses de HS, (5 e 6) Avaliação em HS, (7 e 8 ) Relação entre HS e HSE, (9) Programas de Treinamento em HS, (10) HS e deficiências sensoriais, (11) HS e deficiência intelectual, (12) HS e Transtornos do Espectro do Autismo, (13) HS e Deficiência física, (14 a 17) Avaliação multimodal de alunos com deficiências, (18 a 21) Elaboração de atividades com recursos da CA para crianças/adolescentes com deficiência e, (22) replicação do IHS-Del-Prette e do questionário. 
Patricia Lorena Quiterio, Leila Regina d'Oliveira de Paula Nunes, Bruna de Lima Camelo, Jenniffer Pires da Silva, Marwin Machay Indio do Brasil do Carmo

\section{Análise de Dados}

1. Questionário Diagnóstico. As respostas foram organizadas em categorias (Bardin, 2010) definidas da seguinte forma: (a) definição de CA; (b) definição de HS; (c) conceitos sobre desempenho e CS; (d) HSE e a pessoa com deficiência e; (e) trocas sociais.

2. Inventário de Habilidades Sociais (IHS-Del Prette, 2011). A avaliação dos dados foi realizada por meio do escore total e escores fatoriais. Em função da indisponibilidade de quatro participantes para as avaliações de seguimento e de modo a maximizar o poder estatístico dos dados, os impactos de curto prazo e de longo prazo da intervenção foram analisados separadamente. Para o efeito de curto prazo, testou-se a hipótese de que as distribuições dos escores obtidos por meio do IHS-Del-Prette para as medidas Pósintervenção são diferentes do que as medidas pré-intervenção, utilizando o teste dos postos sinalizados de Wilcoxon (Navarro, 2018). Para a avaliação do efeito de longo prazo foi realizado o teste não paramétrico de Friedman das diferenças entre as medidas repetidas (pré, Pós-intervenção e seguimento). Testes post hoc foram realizados entre todas as condições utilizando o teste de Conover com a correção de Holm. Um fator de nível de significância de 5\% foi utilizado para todas as comparações estatísticas. As análises estatísticas foram conduzidas com auxílio do software de computação estatística R (R Core Team, 2018).

3. Diário de Campo e Filmagens. Realizaram-se 18 vivências e 12 dinâmicas. As filmagens foram analisadas por duas assistentes de pesquisa, não participantes do PPHS. Essa análise foi realizada para cada encontro de intervenção para avaliar a confiabilidade pelo critério de equivalência, isto é, a concordância entre os observadores. Estabeleceu-se o índice de consenso entre as assistentes de pesquisa $\geq 80 \%$ para cada item avaliado, sendo que aqueles que não obtiveram consenso foram discutidos em reunião presencial como forma de contribuir para a discussão dos resultados.

\section{Resultados e Discussão}

O presente estudo buscou avaliar os efeitos de um Programa de Promoção de Habilidades Sociais com foco na inclusão, voltado para estudantes universitários do curso de Pedagogia, a partir de um questionário diagnóstico, de inventário de auto relato e do uso de registros de diário de campo. Os achados são apresentados e discutidos quanto às categorias de análise de conteúdo e por meio de estatística não paramétrica possibilitando uma análise dos aspectos comportamentais e interpessoais das graduandas. 
1. Questionário Diagnóstico (Pré- e Pós-intervenção). As graduandas possuíam percepção satisfatória dos conceitos. Contudo, ressalta-se que houve uma ampliação dos conceitos, de sua aplicabilidade e, essencialmente, do papel do interlocutor na promoção das HS.

Categoria 1: Definição de CA - observou-se que as graduandas - nos questionários inicial e final - possuíam informações sobre conceitos teóricos, usuários e sistemas de comunicação que envolvem a área da CA, provavelmente devido ao fato de cursarem disciplinas que abordavam questões relacionadas à área. Parte das graduandas (80\%) focava na CA como um recurso para o indivíduo: "É um meio em que os deficientes usam para se comunicar" (P8) e após a intervenção, pode-se ampliar o entendimento da funcionalidade dos recursos da CA, bem como a importância do interlocutor e a possibilidade de favorecer a comunicação e possibilitar a autonomia (90\%): “(...) utilizando símbolos gráficos, expressando desejos, necessidades e sentimentos, tornando o indivíduo mais independente" (P8) "de forma que as mesmas possuam um canal de comunicação com o meio" (P2). Essa ampliação do conceito vai ao encontro da literatura (Quiterio \& Nunes, 2013), pois os recursos são importantes, mas é essencial que se ofereça acesso à comunicação e interação efetivas.

Categoria 2: Definição de HS - parte das graduandas (60\%), inicialmente, definiu as HS como um conjunto de comportamentos: "É um conjunto de ações" (P2) e uma participante (P7) colocou não ter noção do conceito. Posteriormente, percebeu-se a ampliação do conceito (90\%), bem como se sinalizou que os comportamentos devem ser adequados e que devem respeitar o limite do outro. Conforme ressaltam Z. A. P. Del Prette e A. Del Prette (2007) os comportamentos emitidos devem ser adequados à situação e devem respeitar esses comportamentos nos outros: "HS são "normas" importantes no relacionamento tanto com o outro como no ambiente, que variam de acordo a cultura em que está inserido" (P2).

Categoria 3: Conceitos sobre desempenho e competência social - parte das graduandas não diferenciava os conceitos (50\%): “Algo ligado à sua relação com o social” (P5). Mas no decorrer da intervenção puderam diferenciá-los e ampliá-los (80\%): "Desempenho social é como a pessoa reage em determinada situação. Já a competência social é o como ela deve se comportar em uma determinada situação, sendo "ensinada" anteriormente" (P5). Essa fala corrobora com a literatura que ressalta a dimensão avaliativa da CS, que se expressa no desempenho, enquanto comportamento observável (A. Del Prette \& Z. A. P. Del Prette, 2017). 
Categoria 4: HSE e a pessoa com deficiência - todas as participantes destacaram a importância das HS para todas as pessoas, incluindo aquelas com deficiência. Contudo, observou-se um predomínio de respostas sinalizando que essas pessoas é que precisam se desenvolver (60\%): "É uma forma de fazer com que eles desenvolvam suas competências sociais" (P4). A literatura indica que as pessoas com deficiência precisam desenvolver as HS (Rosin-Pinola et al., 2017), mas enfatiza a necessidade de atentar para os interlocutores, fundamentais neste processo, pois para exercitar as HS é preciso um parceiro de comunicação (Quiterio \& Nunes, 2017). Ao final da intervenção, observou-se uma reflexão sobre este aspecto (100\%): "Porque ao entender as HS podemos desenvolver junto com as pessoas com deficiência uma relação mais eficaz e inseri-las na sociedade, fazendo com que elas não sejam excluídas" (P4). Alguns estudos (Manolio, 2009; Rosa \& Menezes, 2019) indicam que os professores não incentivam situações de interação entre aluno com deficiência e seus colegas, e as crianças não demonstram iniciativa de contato nas relações interpessoais. Deste modo, ao investir na formação inicial, os professores podem ser capazes de utilizar a interação social, possibilitando reações adequadas e modelos socialmente competentes.

Categoria 5: Trocas sociais - as graduandas reconheceram a importância da interação social: “As HS são estabelecidas, aprendidas na relação com o outro" (P6). Ao final, observou-se novamente uma ampliação dos conceitos (90\%): “O homem é um ser social e precisa interagir com o outro [...] Deficientes ou não, somos todos dependentes" (P6). Nas trocas sociais há necessidade de os indivíduos estarem disponíveis, desejosos de acolher e responder às mensagens do indivíduo com deficiência, oferecendo condições para maximizar a autonomia comunicativa de seu interlocutor sem fala articulada (Quiterio \& Nunes, 2013).

\section{Inventário de Habilidades Sociais (IHS-Del Prette, 2011) (Pré-, Pós-intervenção e}

Seguimento). Na análise dos efeitos de curto prazo, para o Escore Total do IHS-Del-Prette, os escores de Pós-intervenção foram significativamente maiores do que os escores préintervenção $(W=2,00, p<0,01, r=-0,625)$. Para o Fator 1 (Enfrentamento e Autoafirmação com risco) os escores de Pós-intervenção também foram significativamente maiores do que os escores pré-intervenção $(W=10,00, p<0,05, r=-0,454)$. De modo semelhante, o Fator 3 (Conversação e desenvoltura social) $(W=7,00, p<0,05, r=-0,526)$, também apresentou mudanças estatisticamente significantes, conforme demonstra a Tabela 1. Por outro lado, para os demais fatores, a análise não indicou diferença significativa entre as medidas. 
Patricia Lorena Quiterio, Leila Regina d'Oliveira de Paula Nunes, Bruna de Lima Camelo, Jenniffer Pires da Silva, Marwin Machay Indio do Brasil do Carmo

\section{Tabela 1}

Resultados do IHS-Del Prette (pré e pós-intervenção)

\begin{tabular}{lll}
\hline Variável & Mediana pré (IIQ) & Mediana pós (IIQ) \\
\hline Escore total & $87,0(76,0-92,5)$ & $98,0(88,8-107)$ \\
F1 & $7,48(6,30-9,13)$ & $9,38(8-10,2)$ \\
F2 & $7,83(7,58-8,89)$ & $9,25(7,82-10,1)$ \\
F3 & $6,28(5,79-7,14)$ & $7,63(1,41)$ \\
F4 & $3,26(2,49-4,13)$ & $3,23(3,15-4,19)$ \\
F5 & $1,02(0,71-1,20)$ & $1,00(0,79-1,41)$ \\
\hline
\end{tabular}

Nota. IIQ = Intervalo interquartil

O Fator 1 - Enfrentamento e autoafirmação com risco - revelou que o grupo apresentou melhoras, após a intervenção, nesses itens que envolvem lidar com situações interpessoais em ambientes de discordância presumida por meio da afirmativa de seus direitos, preservando sua autoestima em situações que apresentem a possibilidade de reação indesejável do interlocutor. Destaca-se a importância das habilidades de falar em público e lidar com críticas, que envolvem, por parte do professor, tanto as relações interpessoais no contexto da sala de aula como no ambiente escolar, na interação com familiares dos alunos, colegas de trabalho e gestores. Este resultado corrobora com outros estudos (Bolsoni-Silva et al, 2009; A. Del Prette \& Z. A. P. Del Prette, 2003; Ferreira et al, 2014; Lopes et al., 2017) nos quais participantes de PPHS ampliaram este fator. Ainda em F1, destaca-se a habilidade de fazer perguntas - no sentido de buscar informação, estratégias e recursos alternativos e, compartilhá-los com alunos e familiares de crianças com e sem deficiência (Quiterio \& Nunes, 2017). As habilidades que compõem o F3 - Conversação e Desenvoltura Social demandam prática social na conversação. Os ganhos estatisticamente significativos do grupo, após a intervenção, podem significar o desenvolvimento satisfatório no traquejo social para iniciar, manter e finalizar uma conversação, o que revela conhecimento das normas de interação social, conforme destacam Chaves et al. (2017). Essas habilidades, como as relacionadas à conversação, devido ao caráter interativo do ambiente escolar, revelam-se necessárias (Manolio, 2009). Outras habilidades abarcadas nesse fator, como pedir favores aos colegas e recusar pedidos abusivos, podem favorecer o estabelecimento de parcerias com diferentes agentes educativos no desenvolvimento de atividades acadêmicas e sociais com foco inclusivo. Ainda em F3, conforme afirma a literatura, desenvolveu-se prática de reagir a um elogio por meio de expressão de afeto positivo e de afirmação da autoestima no contexto 
Patricia Lorena Quiterio, Leila Regina d'Oliveira de Paula Nunes, Bruna de Lima Camelo, Jenniffer Pires da Silva, Marwin Machay Indio do Brasil do Carmo

diário (Z. A. P. Del Prette \& A. Del Prette, 2007). Atenta-se para o fato de que em revisão de estudos recentes com estudantes da área de ciências humanas foram encontrados resultados semelhantes (Leme et al, 2016). Os resultados, a partir do IHS-Del Prette (Z. A. P. Del Prette \& A. Del Prette, 2011), sugerem que houve uma melhora no desenvolvimento das HS de todas as alunas, o que pode interferir de modo satisfatório em sua futura atividade docente.

Segundo Ferreira et al. (2014), a estratégia de seguimento demonstra que o impacto da replicação dos instrumentos após o processo interventivo mostra-se satisfatório na análise dos resultados. Neste estudo, o seguimento com as graduandas foi conduzido dois anos após a conclusão do PPHS. A Tabela 2 apresenta a análise comparativa entre pré-, Pós-intervenção e seguimento das seis graduandas. Os resultados do IHS-Del-Prette revelaram que houve uma manutenção nas habilidades, comparado ao pós-teste, que constituem cada subclasse de HS.

\section{Tabela 2}

Resultados do IHS-Del Prette (pré-, Pós-intervenção e seguimento)

\begin{tabular}{lccc}
\hline Variável & Pré-intervenção $(n=6)$ & Pós-intervenção $(n=6)$ & Seguimento $(n=6)$ \\
\cline { 2 - 4 } & Mediana (IIQ) & Mediana (IIQ) & Mediana (IIQ) \\
\cline { 3 - 5 } IHS total & $85,0(76,0-92,5)$ & $103(89,9-110)$ & $107(101-119)$ \\
F1 & $6,91(9,3-5,57)$ & $9,38(8,76-10,1)$ & $10,6(10,3-12,5)$ \\
F2 & $7,75(7,58-8,77)$ & $9,36(8,35-10,6)$ & $10,5(9,99-10,6)$ \\
F3 & $6,79(6,00-7,14)$ & $7,53(6,89-8,40)$ & $8,46(7,89-8,81)$ \\
F4 & $3,75(3,23-4,23)$ & $3,75(3,20-4,78)$ & $4,18(4,00-4,89)$ \\
F5 & $1,17(0,96-1,20)$ & $1,05(0,85-1,47)$ & $1,21(1,17-1,45)$ \\
\hline
\end{tabular}

Nota. IIQ = Intervalo interquartil

As HS das participantes foram avaliadas em três condições (pré, Pós-intervenção e seguimento). Para que o teste de Friedman fosse realizado, os dados de quatro participantes que não realizaram o seguimento foram retirados para atender à exigência de balanceamento de observações entre os grupos. Foram observadas diferenças estatisticamente significantes entre as condições, no Escore Total do IHS, $\chi^{2}(2)=10,33, p<0,01$, nos fatores, Enfrentamento com risco, $\chi^{2}(2)=12,00, p<0,01$ e Autoafirmação na expressão de afeto positivo, $\chi^{2}(2)=6,35, p<0,05$. Foram conduzidos testes post hoc de Conover com aplicação da correção de Holm. Os resultados indicaram diferenças estatisticamente significantes para os escores no seguimento, em relação à pré-intervenção, no Escore Total $(p>0,05)$ e no fator Enfrentamento com risco $(p>0,05)$. 
Patricia Lorena Quiterio, Leila Regina d'Oliveira de Paula Nunes, Bruna de Lima Camelo, Jenniffer Pires da Silva, Marwin Machay Indio do Brasil do Carmo

Pode-se constatar que a melhora nos fatores de HS se manteve em médio prazo. Não ocorreram oscilações significativas desses resultados após dois anos de curso, indicando estabilidade nos resultados.

3. Diário de Campo (Avaliação Processual). A análise desse instrumento contribuiu para o acompanhamento do processo por meio dos registros realizados acerca do PPHS e das vivências propostas. Em uma das primeiras vivências, pode-se constatar uma tendência em minimizar o positivo, acreditando que não seriam capazes de completar a tarefa, bem como a preocupação com a opinião do outro: "Quando eu dei o comando vocês acharam que iriam fazer dez rodadas? (Facilitadora) / Não, duas com certeza! (P4) / Na atividade a gente tinha que ouvir a opinião dos outros né, eles estão me subestimando?! (P8)”. Um ambiente que promova saúde mental faz-se necessário para o desenvolvimento de habilidades cognitivas, emocionais e interpessoais (Bolsoni-Silva et al., 2013; Esteves, 2018).

Na vivência "Uma viagem à lua" (Weber, 2011), P6 fez o seguinte elogio: "Vou declarar a minha amizade a P3 que entrou na nossa turma esse período e tá sendo maravilhosa a presença dela e tá sendo muito enriquecedor pra gente". Fazer elogios é uma habilidade importante no relacionamento interpessoal (Reis et al, 2012; Rosa \& Menezes, 2019).

No encontro seguinte, durante a vivência: "Corredor brasileiro" (Z. A. P. Del Prette \& A. Del Prette, 2007) a maioria das graduandas sinalizou maior dificuldade na subclasse assertividade e, algumas acrescentaram a habilidade de falar em público. Isto é, identificou-se o déficit decorrente da dificuldade de negar pedidos de modo adequado (Chaves et al, 2017).

No sétimo encontro, com foco nas HSE que o professor precisa desenvolver para propiciar a aprendizagem tanto acadêmica quanto interpessoal (Manolio, 2009; Merritt et al, 2012; Z. A. P. Del Prette \& A. Del Prette, 2007), ao receber a frase com comentários não adequados sobre seu desempenho e marcar em uma folha a emoção que a frase lhe despertou, P5, referindo-se a comparações feitas por um professor, disse: "Essas comparações não são muito legais, porque a pessoa que não tá sendo elogiada, não tá sendo exaltada pelas suas habilidades, pelas suas aptidões fica se sentindo meio...". Ressaltou-se como as atitudes e falas dos professores refletem no comportamento do aluno, podendo reverberar ao longo de sua vida. Para Manolio (2009), o professor precisa estabelecer contatos sociais adequados e atentar para seu papel como modelo competente como educador. Durante o debate, a habilidade de fazer e receber elogios foi novamente ressaltada: “às vezes a gente não tá muito preparado pra receber aquele elogio, às vezes eu fico meio constrangida assim e tal, mas com o tempo você vai sabendo lidar aí, é melhor receber um elogio e também é legal você fazer um elogio da mesma forma que não vai constranger e nem minimizar os outros que estão 
presentes" (P5). Na segunda etapa da atividade, ao receber o par da sua frase, sendo agora uma sentença socialmente habilidosa, as graduandas perceberam que a maneira de falar com os alunos causa consequências diferenciadas, como disse P5: “(...) na escola acontece muito isso, a criança acaba sendo estigmatizada".

$\mathrm{Na}$ aula nove do PPHS, na qual deveriam assumir um lugar numa fila em ordem crescente, mas com olhos vendados e sem utilizar a linguagem verbal, as alunas comunicaram-se por meio das mãos e do toque para descobrir a sequência e formar a fila. Diferentes estratégias foram pensadas, evidenciando o trabalho em equipe para a resolução do problema. A vivência trouxe discussão sobre persistência, resolução de problemas, autocontrole, linguagem não verbal e trabalho em equipe: "no trabalho de equipe é mais construtivo [...] tem sempre que depender de alguém pra construir alguma coisa" (P3). O desenvolvimento e uso dessas estratégias são importantes para o relacionamento interpessoal, inclusive, no ambiente escolar (Rosin-Pinola \& Del Prette, 2014). E, no décimo encontro do curso, na vivência "Reconhecendo e comunicando emoções" (A. Del Prette \& Del Prette, 2007), a discussão focou na necessidade de envolvimento do interlocutor na interação, possibilitando a comunicação adequada entre os pares (Quiterio \& Nunes, 2013).

Pode-se constatar que, no início, havia certo distanciamento entre as alunas, minimizado no decorrer do PPHS. Os resultados evidenciaram a importância de desenvolver as HS, incluindo o aspecto não verbal, especialmente no caso dessa intervenção voltada para a inclusão de pessoas com deficiência. Por fim, criou-se o sentimento de aceitação e pertencimento no grupo, promoveu-se a resolução de problemas e a prática de fazer/receber elogios, o que aumenta a probabilidade de generalização do reforçamento positivo em outros contextos.

\section{Considerações Finais}

Graduandos em Pedagogia participaram de estudos que envolveram avaliação das HS (Ferreira et al. 2014) e participaram igualmente de PPHS em estudos sobre a formação continuada do professor (Cintra, 2018; Manolio, 2009; Rosin-Pinola et al., 2017). Contudo, não foi localizado estudo voltado para formação inicial de graduandos em Pedagogia.

A proposta foi oferecer uma formação em HS e influenciar positivamente o desenvolvimento das HSE de modo que as futuras professoras pudessem refletir sobre algumas questões: Como posso desenvolver as HS dos alunos se não conheço as minhas dificuldades e potencialidades interpessoais? Como desenvolver as HSE? Como aproveitar 
Patricia Lorena Quiterio, Leila Regina d'Oliveira de Paula Nunes, Bruna de Lima Camelo, Jenniffer Pires da Silva, Marwin Machay Indio do Brasil do Carmo

situações de sala de aula para promover as HS? Como propiciar a interação entre alunos com e sem deficiência? Neste sentido, as graduandas puderam discutir e buscar conhecimento sobre cada um destes aspectos, bem como elaborar estratégias de acordo com o contexto e os alunos. Considera-se que o desenvolvimento de PPHS seja uma ferramenta valiosa em todos os níveis de atuação em saúde e educação, sendo útil para minimizar fatores de risco e incrementar fatores de proteção ao desenvolvimento humano, tratar problemas já instalados e reduzir o impacto de déficits em HS (Z. A. P. Del Prette \& A. Del Prette, 2012).

Em relação às práticas educativas, percebeu-se que as graduandas, apesar do conhecimento adquirido na formação acadêmica, direcionavam ao aluno a responsabilidade sobre dificuldades, bem como acreditavam que os recursos, por si só, poderiam resolver os déficits comunicativos. Este dado inicial demonstra a tendência da sociedade de classificar a pessoa com deficiência de acordo com o modelo biomédico (Deimling, 2013). Neste sentido, buscou-se desenvolver junto às graduandas posturas, reações e estratégias que propiciassem a inclusão destes alunos como sujeitos participantes da sociedade. Ao final do PPHS, constatouse uma mudança de percepção como pessoas fundamentais na parceria educativa, compreendendo e exercitando o papel de interlocutor que promove a inserção comunicativa e social (Quiterio \& Nunes, 2013).

As tarefas de casa, bem como as vivências usadas durante o PPHS, foram vistas como positivas pelas alunas, permitindo a prática de habilidades importantes na atuação profissional, tais como o planejamento e execução de dinâmicas grupais. A qualidade dos trabalhos desenvolvidos com recursos da CA sugeriram ganhos em conhecimentos teóricos e práticos de como elaborar materiais para o aluno com deficiência.

Destaca-se que os fatores de assiduidade, presença e participação contribuíram para a efetividade do programa. Os relatos das graduandas no final do curso apontaram também para algumas mudanças no repertório pessoal de HS: "Eu não sei, eu acho que eu sou outra Debora" (nome fictício - P3). Além disso, algumas reações demonstram mudanças nas atitudes das graduandas, como: (a) iniciar, manter e encerrar conversação; (b) lidar com críticas justas e injustas; (c) pedir ajuda/favores; (d) recusar pedido abusivo; (e) lidar com chacotas e; (f) elogiar e agradecer elogios. Estes aspectos são fundamentais no contexto da sala de aula, pois o professor é essencial como modelo de conduta que favoreça o respeito, inclusive utilizando o reforçamento positivo, que produz mudança satisfatória de comportamento (Quiterio \& Nunes, 2013). Educadores podem contribuir para o desenvolvimento da CS, especialmente dos alunos com deficiência (Manolio, 2009; RosinPinola et al., 2017). O futuro educador, desde a graduação, precisa aprender a aproveitar e a 
planejar situações que promovam a comunicação e a interação social no contexto escolar. As atividades em sala de aula fornecem oportunidades de comunicação entre pares, exigindo preparação adicional pelo professor, que pode atuar como multiplicador da promoção das HS (Quiterio \& Nunes, 2017).

Os ganhos das alunas foram revelados pela avaliação Pós-intervenção e da manutenção das mudanças significativas no seguimento. Essas mudanças contribuíram para uma formação acadêmica e profissional, favorecendo o desenvolvimento de diferentes competências, dentre elas a competência teórica, técnica, ética e social. A CS é requerida pelos futuros professores para atuação em qualquer campo profissional, especialmente em sala de aula, como principais preditores de mudança. Uma inclusão formal e planejada de uma disciplina na formação acadêmica auxiliaria no desenvolvimento das HS em diferentes contextos. Assim, a formação acadêmica e profissional procurou ensinar as habilidades e conhecimentos necessários para o estabelecimento de mecanismos de inclusão nas escolas especiais ou regulares, públicas ou particulares. Além do aspecto acadêmico, intervenções voltadas para as interações sociais podem promover a CS em alunos com deficiência e deve ser uma parte de cada currículo em sala de aula (Wolstencroft et al., 2018). A pesquisa constituiu-se como experiência inovadora de formação inicial de estudantes de Pedagogia na área de HS. O avanço significativo que as graduandas obtiveram possivelmente influenciará positivamente seus relacionamentos interpessoais em quaisquer âmbitos sociais, mas especialmente na educação de pessoas com deficiência, possibilitando um olhar diferenciado e uma prática inclusiva.

O estudo apresentou limitações, como um número reduzido de participantes e a falta de combinação com outras medidas de avaliação, como o grupo controle. Os indicadores de processo e de avaliação dos efeitos dessa intervenção permitiram delinear algumas contribuições para futuros estudos: inserção do grupo controle e intervenções em paralelo com os familiares e as crianças/adolescentes para possibilitar um aprimoramento dos modelos de intervenção. 
Patricia Lorena Quiterio, Leila Regina d'Oliveira de Paula Nunes, Bruna de Lima Camelo, Jenniffer Pires da Silva, Marwin Machay Indio do Brasil do Carmo

\section{Referências}

Bardin, L. (2010). Análise de Conteúdo. Lisboa: Edições 70. (Obra original publicada em 1977)

Bolsoni-Silva, A. T., Leme, V. B. R., Lima, A. M. A., Junior, F. M. C., Correia, M. R. G. (2009). Avaliação de um Treinamento de Habilidades Sociais (THS) com Universitários e Recém Formados. Interação em Psicologia, 13(2), 241-251. doi: 10.5380/psi.v13i2.13597

Bolsoni-Silva, A. T, Mariano, M. L., Loureiro, S. R., \& Bonaccorsi, C. (2013). Contexto escolar: Práticas educativas do professor, comportamento e habilidades sociais infantis. Psicologia Escolar e Educacional, 17(2), 259-269. doi: 10.1590/S141385572013000200008

Chaves, N. M., Galinha, S. M. G. A., \& Gontijo, S. (2017). Formação docente e habilidades sociais: Contribuições para a formação integral de estudantes universitários. Revista da UIIPS, 5(3), 99-114. Recuperado de http://hdl.handle.net/10400.15/1967

Cintra, A. B. (2018). Programa semipresencial de habilidades sociais para professores: Características dos cursistas e indicadores de processo e resultado (Dissertação de mestrado). Universidade Federal de São Carlos, São Carlos, SP, Brasil. Recuperado de https://repositorio.ufscar.br/handle/ufscar/10581

Deimling, N. N. M. (2013). A Educação Especial nos cursos de Pedagogia: Considerações sobre a formação de professores para a inclusão escolar. Revista Educação Unisinos, 17(3), 22-27. doi: 10.4013/edu.2013.173.08

Del Prette, A, \& Del Prette, Z. A. P. (2003). No contexto da travessia para o ambiente de trabalho: Treinamento de habilidades sociais com universitários. Estudos de Psicologia, 8(3), 413-420. doi: 10.1590/S1413-294X2003000300008

Del Prette, A., \& Del Prette, Z. A. P. (2017). Competência Social e Habilidades Sociais: Manual teórico-prático. Petrópolis: Vozes.

Del Prette, Z. A. P., \& Del Prette, A. (2007). Psicologia das habilidades sociais: Terapia e educação. Petrópolis: Vozes.

Del Prette, Z. A. P., \& Del Prette, A. (2012). Social skills and behavior analysis: Historical proximity and new issues. Perspectivas em Análise do Comportamento, 1, 104-115 Recuperado de: http://www.rihs.ufscar.br/wp-content/uploads/2015/10/Social-skillsand-behavior-analysis-Historical-proximity-and-new-issues.pdf 
Del Prette, Z. A. P., \& Del Prette, A. (2011). Inventário de Habilidades Sociais (IHS-DelPrette) (4a ed.). São Paulo: Casa do Psicólogo.

DiPerna, J. C., Lei, P. W., Cheng, W., Hart S. C., \& Bellinger, J. M. (2017). A Cluster Randomized Trial of the Social Skills Improvement System-Classwide Intervention Program (SSIS-CIP) in First Grade. Journal of Educational Psychology, 110(1), 1-16. doi: 10.1037/edu0000191

Esteves, M. M. (2018). Habilidades Sociais e Qualidade de Vida de Professores do Ensino Fundamental (Dissertação de mestrado). Universidade Federal de São Carlos, São Carlos, SP, Brasil. Recuperado de: https://repositorio.ufscar.br/handle/ufscar/9807

Ferreira, V. S., Oliveira, M. A., \& Vandenberghe, L. (2014). Efeitos a curto e longo prazo de um grupo de desenvolvimento de habilidades sociais para universitários. Psicologia: Teoria e Pesquisa, 30(1), 73-81. doi: 10.1590/S0102-37722014000100009

Leme, V. B. R., Del Prette, Z. A. P., \& Del Prette, A. (2016). Habilidades sociais de estudantes de psicologia: Estado da arte no Brasil. In A. B. Soares, L. Mourão \& M. M. P. E. Mota (Eds.), O estudante universitário brasileiro: Características cognitivas, habilidades relacionais e transição para o mercado de trabalho (pp.127-142). Curitiba, PR: Appris.

Linder, J. D. (2008). Maintenance of social skills training of early elementary students. California State University: Fresno.

Lopes, D. C., Dascanio, D., Ferreira, B. C., Del Prette, Z. A. P., \& Del Prette, A. (2017). Treinamento de Habilidades Sociais: Avaliação de um Programa de Desenvolvimento Interpessoal Profissional para Universitários de Ciências Exatas. Interação em Psicologia 21(1), 55-65. doi: 10.5380/psi.v21i1.36210

Manolio, C. L. (2009). Análise das habilidades sociais educativas na interação professoraluno (Dissertação de mestrado). Universidade Federal de São Carlos, São Carlos, SP, Brasil. Recuperado de: https://repositorio.ufscar.br/handle/ufscar/3017

Merritt, E. G., Wanless, S. B., Rimm-Kauffman, S. E., \& Cameron, C. (2012). The Contribution of Teachers' Emotional Support to Children's Social Behaviors and SelfRegulatory Skills in First Grade. School Psychology Review, 41(2), 141-159. doi: $10.1080 / 02796015.2012 .12087517$

Navarro, D. (2018). Learning statistics with R: A tutorial for psychology students and other beginners: (version 0.6). Sydney: University of New South Wales.

Quiterio, P. L., \& Nunes, L. R. O. P. (2017). Formação de professores em habilidades sociais educativas e inclusivas. São Paulo: Memnon. 
Quiterio, P. L., \& Nunes, L. R. O. P. (2013). Desenvolvimento de habilidades sociais de futuros professores no processo de inclusão de alunos com deficiência. In R. Glat \& M. Pletsh (Eds.), Estratégias Educacionais Diferenciadas para alunos com necessidades especiais (pp. 107-126). Rio de Janeiro, RJ: EdUERJ.

R Core Team (2018). R: A language and environment for statistical computing. Vienna, Austria: R Foundation for Statistical Computing. Recuperado de https://www.Rproject.org/

Reis, V. T. C., Prata, M. A. R., \& Soares, A. B. (2012). Habilidades sociais e afetividade no contexto escolar: Perspectivas envolvendo professores e ensino-aprendizagem. Psicologia Argumento, 30(69), 347-357. doi: 10.7213/psicolargum.v30i69.23290

Rosa, L. R., \& Menezes, A. B. (2019). Inclusión Escolar e Interacción Social: Una Revisión de Literatura. Trends in Psychology, 27(2), 385-400. doi: 10.9788/tp2019.2-07

Rosin-Pinola, A. R., \& Del Prette, Z. A. P. (2014). Inclusão escolar, formação de professores e a assessoria baseada em habilidades sociais educativas. Revista Brasileira de Educação Especial, 20(3), 341-356. doi: 10.1590/S1413-65382014000300003

Rosin-Pinola, A. R., Marturano, E. M., Elias, L. C. S., \& Del Prette, Z. A. P. (2017). Ensinando habilidades sociais educativas para professores no contexto da inclusão escolar. Revista Educação Especial, 30(59), 737-749. doi: 10.5902/1984686X28430

Vieira-Santos, J., Del Prette, Z. A. P., \& Del Prette, A. (2018). Habilidades sociais educativas: Revisão sistemática da produção brasileira. Avances en Psicología Latinoamericana, 36(1), 45-63. doi: 10.12804/revistas.urosario.edu.co/apl/a.5069

Vila, E. M. (2019). Efeitos de um Programa Parental em Grupo para Mães de Crianças com Problemas de Comportamento: Um Estudo Experimental (Tese de Doutorado). Programa de Pós Graduação em Educação, Universidade Federal do Paraná, Curitiba, Brasil. Recuperado de https://acervodigital.ufpr.br/handle/1884/62490

Weber, L., Salvador, A. P., \& Brandenburg, O. (2011). Programa de Qualidade na Interação Familiar: Manual para Aplicadores (2a ed.). Curitiba: Juruá.

Wolstencroft, J., Robinson, L., Srinivasan, R., Kerry, E., Mandy, W., \& Skuse, D. (2018). A Systematic Review of Group Social Skills Interventions, and Meta-analysis of Outcomes, for Children with High Functioning ASD. Journal Of Autism And Developmental Disorders, 48(7), 2293-2307. doi: 10.1007/s10803-018-3485-1 
Patricia Lorena Quiterio, Leila Regina d'Oliveira de Paula Nunes, Bruna de Lima Camelo, Jenniffer Pires da Silva, Marwin Machay Indio do Brasil do Carmo

\section{Endereço para correspondência}

\section{Patricia Lorena Quiterio}

Rua São Francisco Xavier, 524, Maracanã, Rio de Janeiro - RJ, Brasil. CEP 20550-900

Endereço eletrônico: patricialorenauerj@gmail.com

\section{Leila Regina d'Oliveira de Paula Nunes}

Rua São Francisco Xavier, 524, Maracanã, Rio de Janeiro - RJ, Brasil. CEP 20550-900

Endereço eletrônico: leilareginanunes@gmail.com

\section{Bruna de Lima Camelo}

Rua São Francisco Xavier, 524, Maracanã, Rio de Janeiro - RJ, Brasil. CEP 20550-900

Endereço eletrônico: blima1095@gmail.com

\section{Jenniffer Pires da Silva}

Rua São Francisco Xavier, 524, Maracanã, Rio de Janeiro - RJ, Brasil. CEP 20550-900

Endereço eletrônico: jennifferpsilva@gmail.com

\section{Marwin Machay Indio do Brasil do Carmo}

Rua São Francisco Xavier, 524, Maracanã, Rio de Janeiro - RJ, Brasil. CEP 20550-900

Endereço eletrônico: marwinbr@gmail.com

Recebido em: 05/03/2020

Reformulado em: 25/07/2020

Aceito em: 28/07/2020

\section{Notas}

* Psicóloga, Pedagoga, Doutora pela Universidade do Estado do Rio de Janeiro. Professora Adjunta do Instituto de Psicologia da Universidade do Estado do Rio de Janeiro (UERJ).

** Psicóloga, Pós-Doutora pela Vanderbilt University (USA). Professora Titular na Faculdade de Educação e no Programa de Pós-Graduação em Educação da Universidade do Estado do Rio de Janeiro (UERJ), Rio de Janeiro, Brasil.

*** Graduanda em Psicologia pela Universidade do Estado do Rio de Janeiro (UERJ). Bolsista de Iniciação Científica, Rio de Janeiro, Brasil.

**** Graduanda em Psicologia pela Universidade do Estado do Rio de Janeiro (UERJ). Bolsista pelo CETREINA (Extensão), Rio de Janeiro, Brasil.

***** Graduando em Psicologia pela Universidade do Estado do Rio de Janeiro (UERJ). Bolsista pela FAPERJ, Rio de Janeiro, Brasil.

Financiamento: a pesquisa relatada no manuscrito foi financiada pela bolsa de doutorado da primeira autora (CAPES/Projetos Especiais, PROESP).

Este artigo de revista Estudos e Pesquisas em Psicologia é licenciado sob uma Licença Creative Commons Atribuição-Não Comercial 3.0 Não Adaptada. 